\title{
COMPARISON OF MULTIGRADED AND UNGRADED COUSIN COMPLEXES
}

\author{
M. H. DOGANI AGHCHEGHLOO ${ }^{1}$, R. ENSHAEI ${ }^{2}$, S. GOTO ${ }^{3}$ AND R. Y. SHARP \\ ${ }^{1}$ Department of Pure Mathematics, Faculty of Mathematics, \\ The University of Shahid Bahonar (Kerman), Kerman, \\ Islamic Republic of Iran (mhdogani@arg3.uk.ac.ir) \\ ${ }^{2}$ Department of Mathematics, University of Isfahan, \\ Isfahan 81744, Islamic Republic of Iran (rensh@math.ui.ac.ir) \\ ${ }^{3}$ Department of Mathematics, School of Science and Technology, \\ Meiji University, Higashi-mita 1-1-1, Tama-ku, \\ Kawasaki-shi 214-8571, Japan (goto@math.meiji.ac.jp) \\ ${ }^{4}$ Department of Pure Mathematics, University of Sheffield, \\ Hicks Building, Sheffield S3 7RH, UK (R.Y.Sharp@sheffield.ac.uk)
}

(Received 29 July 1999)

\begin{abstract}
This paper generalizes, in two senses, work of Petzl and Sharp, who showed that, for a $\mathbb{Z}$ graded module $M$ over a $\mathbb{Z}$-graded commutative Noetherian ring $R$, the graded Cousin complex for $M$ introduced by Goto and Watanabe can be regarded as a subcomplex of the ordinary Cousin complex studied by Sharp, and that the resulting quotient complex is always exact. The generalizations considered in this paper are, firstly, to multigraded situations and, secondly, to Cousin complexes with respect to more general filtrations than the basic ones considered by Petzl and Sharp. New arguments are presented to provide a sufficient condition for the exactness of the quotient complex in this generality, as the arguments of Petzl and Sharp will not work for this situation without additional input.
\end{abstract}

Keywords: $\mathbb{Z}^{n}$-graded commutative Noetherian ring; Cousin complex; local cohomology modules

AMS 2000 Mathematics subject classification: Primary 13A02; 13E05; 13D25; 13D45

\section{Introduction}

In [6], Petzl and Sharp compared the graded Cousin complex ${ }^{*} C(M)^{\bullet}$ (introduced by Goto and Watanabe in [4]) for a graded module $M$ over a $\mathbb{Z}$-graded commutative Noetherian ring ( $\mathbb{Z}$ denotes the set of integers) with the ordinary Cousin complex $C(M)^{\bullet}$ (studied by Sharp in $[\mathbf{7}])$ obtained by ignoring the gradings. The main results of $[\mathbf{6}]$ are that ${ }^{*} C(M)^{\bullet}$ can be viewed as a subcomplex of $C(M)^{\bullet}$, and that the resulting quotient complex is always exact.

This paper is concerned with generalizations of that work to multigraded situations and to more general Cousin complexes. 
Throughout, let $G$ be a finitely generated free abelian group, and let $R$ be a $G$-graded commutative Noetherian ring. Let $M$ be a $G$-graded $R$-module. In [5, Chapter I, $\S 4$ ], Goto and Watanabe introduced the $G$-graded Cousin complex ${ }^{*} C(M)^{\bullet}$ for $M$. It is natural to ask whether the analogues of the results of $[\mathbf{6}]$ hold in this $G$-graded situation. One of the aims of this paper is to show that they do; however, while the fact that ${ }^{*} C(M)^{\bullet}$ can be viewed as a subcomplex of the ungraded Cousin complex $C(M)^{\bullet}$ can be proved by straightforward modification of arguments in [6], we needed fresh ideas to prove, in this $G$-graded case, that the resulting quotient complex is always exact, as the argument in [6] does not deal with the case where $\operatorname{rank} G>1$.

The other way in which this paper generalizes work from $[\mathbf{6}]$ is that here we consider the Cousin complex $C(\mathcal{F}, M)^{\bullet}$ with respect to a general filtration $\mathcal{F}$ of $\operatorname{Spec}(R)$ which admits $M$, as in $[\mathbf{8}, \S 1]$ : in this situation, it turns out that there is a natural way to construct a $G$ graded Cousin complex ${ }^{*} C\left({ }^{*} \mathcal{F}, M\right)^{\bullet}$ which can be regarded as a subcomplex of $C(\mathcal{F}, M)^{\bullet}$; we provide conditions on $M$ and $\mathcal{F}$ that are sufficient for the resulting quotient complex to be exact, and show by example that, even in the case where $G=\mathbb{Z}$, the quotient complex need not be exact.

\section{Partial small supports}

In this section we introduce a refinement of the concept of small support (see [6, Definition 1.1]) of a module over a commutative Noetherian ring. We shall make substantial use of this refinement.

Definition 2.1. Let $L$ be a module over the commutative Noetherian ring $A^{\prime}$. For each $i \in \mathbb{N}_{0}$, the $i$-th partial small support (or $i$-th small support) of $L$, denoted by $\operatorname{supp}^{i}(L)$ or $\operatorname{supp}_{A^{\prime}}^{i}(L)$, is defined by

$$
\begin{aligned}
\operatorname{supp}^{i}(L) & =\left\{\mathfrak{p} \in \operatorname{Spec}\left(A^{\prime}\right): \operatorname{Ext}_{A_{\mathfrak{p}}^{\prime}}^{i}\left(k(\mathfrak{p}), L_{\mathfrak{p}}\right) \neq 0\right\} \\
& =\left\{\mathfrak{p} \in \operatorname{Spec}\left(A^{\prime}\right): \mu^{i}(\mathfrak{p}, L)>0\right\},
\end{aligned}
$$

where $k(\mathfrak{p})=A_{\mathfrak{p}}^{\prime} / \mathfrak{p} A_{\mathfrak{p}}^{\prime}$ and $\mu^{i}(\mathfrak{p}, L)$ denotes the $i$ th Bass number of $L$ with respect to $\mathfrak{p}$. Note that the small support $\operatorname{supp}(L)$ of $L$ (see [6, Definition 1.1]) is given by

$$
\operatorname{supp}(L)=\bigcup_{i \in \mathbb{N}_{0}} \operatorname{supp}^{i}(L) .
$$

The next lemma is a refinement of [6, Lemma 1.2]; it can be proved by similar arguments to those used by Petzl and Sharp in [6], and so it is just stated here without proof. The properties of partial small supports presented in the lemma are central to our argument in $\S 4$.

Lemma 2.2. Let $L$ be a module over the commutative Noetherian ring $A^{\prime}$ and let

$$
0 \longrightarrow L^{\prime} \longrightarrow M^{\prime} \longrightarrow N^{\prime} \longrightarrow 0
$$

be a short exact sequence of $A^{\prime}$-modules and $A^{\prime}$-homomorphisms. Let $i \in \mathbb{N}_{0}$. Then 
(i) $\operatorname{Ass}(L)=\operatorname{supp}^{0}(L)$;

(ii) if $S$ is a multiplicatively closed subset of $A^{\prime}$ and $N$ is an $S^{-1} A^{\prime}$-module,

$$
\operatorname{supp}_{A^{\prime}}^{i}(N)=\left\{\mathfrak{p} \in \operatorname{Spec}\left(A^{\prime}\right): \mathfrak{p} \cap S=\emptyset \text { and } \mathfrak{p} S^{-1} A^{\prime} \in \operatorname{supp}_{S^{-1} A^{\prime}}^{i}(N)\right\} ;
$$

(iii) $\operatorname{supp}^{i}\left(M^{\prime}\right) \subseteq \operatorname{supp}^{i}\left(L^{\prime}\right) \cup \operatorname{supp}^{i}\left(N^{\prime}\right)$;

(iv) $\operatorname{supp}^{i}\left(N^{\prime}\right) \subseteq \operatorname{supp}^{i}\left(M^{\prime}\right) \cup \operatorname{supp}^{i+1}\left(L^{\prime}\right)$; and

(v) $\operatorname{supp}^{i}\left(L^{\prime}\right) \subseteq \operatorname{supp}^{i}\left(M^{\prime}\right) \cup \operatorname{supp}^{i-1}\left(N^{\prime}\right)$ (we interpret $\operatorname{supp}^{i-1}\left(N^{\prime}\right)=\emptyset$ if $i=0$ ).

The results of [6, Lemma 1.2] are an immediate consequence of Lemma 2.2.

\section{Multigraded Cousin complexes}

Notation 3.1. Throughout the paper, $G$ will denote a finitely generated free abelian group, and $M$ will denote a $G$-graded module over the $G$-graded commutative Noetherian ring $R$. Let ${ }^{*} \operatorname{Spec}(R)$ denote the set of all $G$-graded prime ideals of $R$ and let ${ }^{*} \operatorname{Supp}(M)$ be the set of $G$-graded prime ideals in the support of $M$. We shall say that an $R$ homomorphism between $G$-graded $R$-modules is homogeneous if it is homogeneous of degree $0 \in G$.

For an arbitrary ideal $\mathfrak{a}$ of $R$, we denote by $\mathfrak{a}^{*}$ the largest $G$-graded ideal of $R$ contained in $\mathfrak{a}$.

We shall only assume that $R$ is *local, that is, has a unique *maximal ideal (a *maximal ideal of $R$ is a maximal member of the set of $G$-graded proper ideals of $R$ ) when this is explicitly made clear; however, the phrase ' $(R, \mathfrak{m})$ is *local' is to be interpreted as meaning that $R$ is ${ }^{*}$ local and $\mathfrak{m}$ is its unique *maximal ideal.

In [3, Chapter III], Enshaei considered $G$-graded analogues of the Cousin complexes studied in [8, Definition 1.3]. We summarize the main points.

Definitions 3.2. A *filtration of ${ }^{*} \operatorname{Spec}(R)$ is a descending sequence $\mathcal{E}=\left(E_{i}\right)_{i \in \mathbb{N}_{0}}$ of subsets of ${ }^{*} \operatorname{Spec}(R)$, so that

$$
{ }^{*} \operatorname{Spec}(R) \supseteq E_{0} \supseteq E_{1} \supseteq \cdots \supseteq E_{i} \supseteq E_{i+1} \supseteq \cdots,
$$

with the property that, for each $i \in \mathbb{N}_{0}$, each member of $\partial E_{i}:=E_{i} \backslash E_{i+1}$ is a minimal member of $E_{i}$ with respect to inclusion. We say that $\mathcal{E}$ admits $M$ if ${ }^{*} \operatorname{Supp}(M) \subseteq E_{0}$.

Now let $\mathcal{E}=\left(E_{i}\right)_{i \in \mathbb{N}_{0}}$ be a ${ }^{*}$ filtration of ${ }^{*} \operatorname{Spec}(R)$ that admits $M$ in the above sense. The Cousin complex ${ }^{*} C(\mathcal{E}, M)^{\bullet}$ for $M$ with respect to $\mathcal{E}$ is a complex of $G$-graded $R$ modules and homogeneous $R$-homomorphisms having the form

$$
0 \stackrel{d^{-2}}{\longrightarrow} M \stackrel{d^{-1}}{\longrightarrow}{ }^{*} M^{0} \longrightarrow \cdots \longrightarrow{ }^{*} M^{i} \stackrel{d^{i}}{\longrightarrow}{ }^{*} M^{i+1} \longrightarrow \cdots,
$$

with, for each $i \in \mathbb{N}_{0}$,

$$
{ }^{*} M^{i}=\bigoplus_{\mathfrak{p} \in \partial E_{i}}\left(\text { Coker } d^{i-2}\right)_{(\mathfrak{p})}
$$


(Here, '(p)' denotes homogeneous localization at $\mathfrak{p}$, that is, fraction formation with respect to the set of homogeneous elements of $R \backslash \mathfrak{p}$.) The homomorphisms in this complex have the following properties: for $m \in M$ and a $G$-graded prime ideal $\mathfrak{p} \in \partial E_{0}$, the component of $d^{-1}(m)$ in $M_{(\mathfrak{p})}$ is $m / 1$; and for $i>0, x \in M^{i-1}$ and $\mathfrak{q} \in \partial F_{i}$, the component of $d^{i-1}(x)$ in $\left(\text { Coker } d^{i-2}\right)_{(\mathfrak{q})}$ is $\pi(x) / 1$, where $\pi:{ }^{*} M^{i-1} \longrightarrow$ Coker $d^{i-2}$ is the natural epimorphism. The fact that such a complex can be constructed follows from obvious modifications of arguments in $[\mathbf{7}, \S 2]$ and $[\mathbf{5}$, Chapter I, $\S 4]$.

Example 3.3. If ${ }^{*} \mathcal{H}(M)$ denotes the $M$-*height filtration $\left({ }^{*} H_{i}\right)_{i \in \mathbb{N}_{0}}$ of ${ }^{*} \operatorname{Spec}(R)$, defined by

$$
{ }^{*} H_{i}=\left\{\mathfrak{p} \in{ }^{*} \operatorname{Supp}(M): \mathrm{ht}_{M} \mathfrak{p} \geqslant i\right\}, \quad \text { for all } i \in \mathbb{N}_{0}
$$

(where $\operatorname{ht}_{M} \mathfrak{q}=\operatorname{dim}_{R_{\mathfrak{q}}} M_{\mathfrak{q}}$ for $\mathfrak{q} \in \operatorname{Supp}(M)$ ), then ${ }^{*} \mathcal{H}(M)$ admits $M$ and the Cousin complex ${ }^{*} C\left({ }^{*} \mathcal{H}(M), M\right)$ for $M$ with respect to ${ }^{*} \mathcal{H}(M)$ is just the Cousin complex ${ }^{*} C(M)^{\bullet}$ for $M$ considered by Goto and Watanabe in [5, Chapter I, $\S 4$ ].

Example 3.4. If $M^{\prime}$ is a module over a commutative Noetherian ring $R^{\prime}$, and we take $G=0$, then we can regard $R^{\prime}$ and $M^{\prime}$ as $G$-graded in an obvious natural way. Then ${ }^{*} \operatorname{Spec}\left(R^{\prime}\right)=\operatorname{Spec}\left(R^{\prime}\right)$, a ${ }^{*}$ filtration $\mathcal{E}$ of ${ }^{*} \operatorname{Spec}\left(R^{\prime}\right)$ which admits $M$ is just a filtration of $\operatorname{Spec}\left(R^{\prime}\right)$ which admits $M$ in the sense of [8, Definitions 1.1], and the Cousin complex ${ }^{*} C(\mathcal{E}, M)^{\bullet}$ for $M$ with respect to $\mathcal{E}$ is just the Cousin complex $C(\mathcal{E}, M)^{\bullet}$ of $[\mathbf{8}$, Definition 1.3].

We now state the basic theorem on which a comparison of Cousin complexes in our $G$ graded case is based. The theorem can be proved by making straightforward modifications to the arguments used to prove [6, Proposition 3.2 and Theorem 3.3], and so we omit the proof.

Theorem 3.5. Let $\mathcal{F}=\left(F_{i}\right)_{i \in \mathbb{N}_{0}}$ be a filtration of $\operatorname{Spec}(R)$ that admits $M$ (see [8, Definitions 1.1]). We can construct the ungraded Cousin complex $C(\mathcal{F}, M)^{\bullet}$ of $[\boldsymbol{8}$, Definition 1.3]. For each $i \in \mathbb{N}_{0}$ we set ${ }^{*} F_{i}=F_{i} \cap{ }^{*} \operatorname{Spec}(R)$. Then ${ }^{*} \mathcal{F}=\left({ }^{*} F_{i}\right)_{i \in \mathbb{N}_{0}}$ is a ${ }^{*}$ filtration of ${ }^{*} \operatorname{Spec}(R)$ which admits $M$ in the sense of 3.2, and so we can construct the Cousin complex ${ }^{*} C\left({ }^{*} \mathcal{F}, M\right)$ of 3.2 .

We write $C(\mathcal{F}, M)^{\bullet}$ as

$$
0 \stackrel{e^{-2}}{\longrightarrow} M \stackrel{e^{-1}}{\longrightarrow} M^{0} \stackrel{e^{0}}{\longrightarrow} M^{1} \longrightarrow \cdots \longrightarrow M^{i} \stackrel{e^{i}}{\longrightarrow} M^{i+1} \longrightarrow \cdots,
$$

and ${ }^{*} C\left({ }^{*} \mathcal{F}, M\right)^{\bullet}$ as

$$
0 \stackrel{d^{-2}}{\longrightarrow} M \stackrel{d^{-1}}{\longrightarrow} M^{0} \stackrel{d^{0}}{\longrightarrow} M^{1} \longrightarrow \cdots \longrightarrow{ }^{*} M^{i} \stackrel{d^{i}}{\longrightarrow}{ }^{*} M^{i+1} \longrightarrow \cdots .
$$

We use 'overlines' to denote natural images, of elements of terms in Cousin complexes, in cokernels of appropriate maps in those complexes.

There is a chain map

$$
\Psi(\mathcal{F}, M)=\left(\psi^{i}\right)_{i \geqslant-2}:{ }^{*} C\left({ }^{*} \mathcal{F}, M\right)^{\bullet} \longrightarrow C(\mathcal{F}, M)^{\bullet}
$$

of complexes of $R$-modules and $R$-homomorphisms such that the following hold. 
(i) The homomorphism $\psi^{-1}: M \longrightarrow M$ is the identity map.

(ii) For all $i \in \mathbb{N}_{0}$ and all $\left(\overline{y_{\mathfrak{p}}} / t_{\mathfrak{p}}\right)_{\mathfrak{p} \in \partial F_{i} \cap^{*} \operatorname{Spec}(R)} \in{ }^{*} M^{i}$,

$$
\psi^{i}\left(\left(\frac{\overline{y_{\mathfrak{p}}}}{t_{\mathfrak{p}}}\right)_{\mathfrak{p} \in \partial F_{i} \cap^{*} \operatorname{Spec}(R)}\right)=\left(\beta_{\mathfrak{p}}\right)_{\mathfrak{p} \in \partial F_{i}},
$$

where, for $\mathfrak{p} \in \partial F_{i}$,

$$
\beta_{\mathfrak{p}}= \begin{cases}\frac{\overline{\psi^{i-1}\left(y_{\mathfrak{p}}\right)}}{t_{\mathfrak{p}}}, & \text { if } \mathfrak{p} \text { is } G \text {-graded }, \\ 0, & \text { if } \mathfrak{p} \text { is not } G \text {-graded }\end{cases}
$$

(In this notation, each $t_{\mathfrak{p}}$ denotes a homogeneous element of $R$ outside $\mathfrak{p}$, and the $y_{\mathfrak{p}}$ denote elements of ${ }^{*} M^{i-1}$; when $i=0$, interpret ${ }^{*} M^{i-1}$ as $M$ itself.)

(iii) All the $\psi^{i}(i \geqslant-1)$ are monomorphisms.

(iv) We have $\psi^{i}\left(\left(\operatorname{Coker} d^{i-2}\right)_{(\mathfrak{p})}\right) \subseteq\left(\operatorname{Coker} e^{i-2}\right)_{\mathfrak{p}}$ for all $\mathfrak{p} \in \partial F_{i} \cap{ }^{*} \operatorname{Spec}(R)$.

(v) For $\mathfrak{p} \in \partial F_{i} \cap{ }^{*} \operatorname{Spec}(R)$, we have

$$
\left(\text { Coker } e^{i-2}\right)_{\mathfrak{p}} / \psi^{i}\left(\left(\text { Coker } d^{i-2}\right)_{(\mathfrak{p})}\right) \cong H_{\mathfrak{p}}^{i}(M)_{\mathfrak{p}} / H_{\mathfrak{p}}^{i}(M)_{(\mathfrak{p})} .
$$

Definition and Remarks 3.6. In the situation of Theorem 3.5, all the constituent homomorphisms in the chain map

$$
\Psi(\mathcal{F}, M)=\left(\psi^{i}\right)_{i \geqslant-2}:{ }^{*} C\left({ }^{*} \mathcal{F}, M\right)^{\bullet} \longrightarrow C(\mathcal{F}, M)^{\bullet}
$$

are monomorphisms. We can therefore regard ${ }^{*} C\left({ }^{*} \mathcal{F}, M\right)^{\bullet}$ as a subcomplex of $C(\mathcal{F}, M)^{\bullet}$ : we denote the quotient complex $C(\mathcal{F}, M) \bullet / \operatorname{Im}(\Psi(\mathcal{F}, M))$ by

$$
Q(\mathcal{F}, M)^{\bullet}: 0 \longrightarrow Q^{0} \stackrel{f^{0}}{\longrightarrow} Q^{1} \longrightarrow \cdots \longrightarrow Q^{i} \stackrel{f^{i}}{\longrightarrow} Q^{i+1} \longrightarrow \cdots
$$

and refer to this as the generalized degradation complex of $M$ with respect to $\mathcal{F}$.

Let $i \in \mathbb{N}_{0}$. Then there is an obvious isomorphism $Q^{i} \cong G^{i} \oplus U^{i}$, where

$$
G^{i}:=\bigoplus_{\mathfrak{p} \in \partial F_{i} \cap * \operatorname{Spec}(R)}\left(\operatorname{Coker} e^{i-2}\right)_{\mathfrak{p}} / \psi^{i}\left(\left(\operatorname{Coker} d^{i-2}\right)_{(\mathfrak{p})}\right)
$$

and

$$
U^{i}:=\bigoplus_{\mathfrak{p} \in \partial F_{i} \backslash * \operatorname{Spec}(R)}\left(\text { Coker } e^{i-2}\right)_{\mathfrak{p}}
$$

Also, for $\mathfrak{p} \in{ }^{*} \operatorname{Supp}(M)$ with $\mathfrak{p} \in \partial F_{i}$, set

$$
Q^{i}(\mathfrak{p}, \mathcal{F}, M):=\left(\text { Coker } e^{i-2}\right)_{\mathfrak{p}} / \psi^{i}\left(\left(\text { Coker } d^{i-2}\right)_{(\mathfrak{p})}\right)
$$


Now $Q^{i}(\mathfrak{p}, \mathcal{F}, M)$ has a natural structure as an $R_{(\mathfrak{p})}$-module, and it follows from Theorem 3.5(v) and [1, Corollary 4.3.3] that, as such,

$$
Q_{R}^{i}(\mathfrak{p}, \mathcal{F}, M) \cong Q_{R_{(\mathfrak{p})}}^{i}\left(\mathfrak{p} R_{(\mathfrak{p})}, \mathcal{F}_{(\mathfrak{p})}, M_{(\mathfrak{p})}\right),
$$

where the filtration $\mathcal{F}_{(\mathfrak{p})}$ is as defined in [8, Definitions 1.1]. Furthermore, we can also conclude from Theorem 3.5(v) and the $G$-graded analogue of [6, Lemma 2.4] that

$$
\operatorname{Supp}_{R_{(\mathfrak{p})}}\left(Q_{R}^{i}(\mathfrak{p}, \mathcal{F}, M)\right) \subseteq\left\{\mathfrak{q} R_{(\mathfrak{p})}: \mathfrak{q} \in \operatorname{Supp}(M), \mathfrak{q} \text { is not } G \text {-graded and } \mathfrak{q}^{*}=\mathfrak{p}\right\}
$$

It will be noted that much of Theorem 3.5 and 3.6 represent straightforward generalizations of work in [6, Proposition 3.2, Theorem 3.3 and Definition and Notation 3.4]. There is an alternative approach to this work, based on generalized Hughes complexes, presented in $[\mathbf{2}]$.

In our investigation, in $\S 4$ below, of conditions under which the generalized degradation complex $Q(\mathcal{F}, M)^{\bullet}$ is exact, straightforward generalization of the work in $[\mathbf{6}]$ is insufficient, on its own, to achieve our goals.

\section{Exactness of the generalized degradation complex}

In this section we shall discuss exactness of the generalized degradation complex of 3.6. First we show by example that it is not always exact.

Example 4.1. Let $\mathbb{C}$ be the complex field and $A=\mathbb{C}[X]$ be the (Cohen-Macaulay) ring of polynomials in the indeterminate $X$ with coefficients in $\mathbb{C}$, graded in the usual way. Let $\mathcal{H}(A)=\left(H_{i}\right)_{i \in \mathbb{N}_{0}}$ be the height filtration of $\operatorname{Spec}(A)$ in the sense of [8, Examples 1.2], so that $H_{0}=\operatorname{Spec}(A), H_{1}=\{(X-\alpha): \alpha \in \mathbb{C}\}$, and $H_{i}=\emptyset$ for all $i \geqslant 2$. Let $\mathcal{F}=\left(F_{i}\right)_{i \in \mathbb{N}_{0}}$ be given by $F_{0}=H_{0}, F_{1}=H_{1}, F_{2}=\{(X-1)\}$, and $F_{i}=\emptyset$ for all $i \geqslant 3$. Then

$$
\operatorname{Spec}(A)=F_{0} \supseteq F_{1} \supseteq F_{2} \supseteq F_{3}=F_{4}=\cdots=\emptyset .
$$

Furthermore, $F_{0} \backslash F_{1}=\{(0)\}, F_{1} \backslash F_{2}=\{(X-\alpha): 1 \neq \alpha \in \mathbb{C}\}$ and $F_{2} \backslash F_{3}=F_{2}=$ $\{(X-1)\}$. Thus, $\mathcal{F}$ is a filtration of $\operatorname{Spec}(A)$.

Then, with the notation of Theorem 3.5 , we have ${ }^{*} F_{0}=\{(0),(X)\}={ }^{*} H_{0},{ }^{*} F_{1}=$ $\{(X)\}={ }^{*} H_{1}$ and ${ }^{*} F_{i}=\emptyset$ for all $i \geqslant 2$. Since $A$ is Cohen-Macaulay, the graded Cousin complex ${ }^{*} C\left({ }^{*} \mathcal{H}(A), A\right)^{\bullet}$ is exact by $\left[\mathbf{4}\right.$, Theorem 1.3.3] and, since ${ }^{*} C\left({ }^{*} \mathcal{F}, A\right)^{\bullet}=$ ${ }^{*} C\left({ }^{*} \mathcal{H}(A), A\right)^{\bullet}$, it follows that the graded Cousin complex ${ }^{*} C\left({ }^{*} \mathcal{F}, A\right) \cdot{ }^{\bullet}$ is exact.

The Cousin complex $C(\mathcal{H}(A), A)^{\bullet}$, which is exact, has the form

$$
0 \longrightarrow A \longrightarrow K \longrightarrow \bigoplus_{\alpha \in \mathbb{C}}(K / A)_{(X-\alpha)} \longrightarrow 0
$$

where $K=Q(A)$ is the quotient field of $A$. Therefore, the map

$$
\xi: K / A \longrightarrow \bigoplus_{\alpha \in \mathbb{C}}(K / A)_{(X-\alpha)},
$$


defined by $\xi(\gamma)=(\gamma / 1)_{\alpha \in \mathbb{C}}$ for all $\gamma \in K / A$, is an isomorphism. The Cousin complex $C(\mathcal{F}, A)^{\bullet}$ has the form

$$
0 \longrightarrow A \longrightarrow K \stackrel{e^{0}}{\rightarrow} \bigoplus_{\alpha \in \mathbb{C} \backslash\{1\}}(K / A)_{(X-\alpha)} \stackrel{e^{1}}{\rightarrow}\left(\operatorname{Coker} e^{0}\right)_{(X-1)} \longrightarrow 0 .
$$

Now $(1 /(X-1))+A$ is a non-zero element in $K / A$, but $((1 /(X-1))+A) / 1=0$ in $(K / A)_{(X-\alpha)}$ for all $\alpha \in \mathbb{C}$ with $\alpha \neq 1$. Therefore, the induced map

$$
\theta: K / A \longrightarrow \bigoplus_{\alpha \in \mathbb{C} \backslash\{1\}}(K / A)_{(X-\alpha)}
$$

(which is defined by $\theta(\gamma)=(\gamma / 1)_{\alpha \in \mathbb{C} \backslash\{1\}}$ for all $\left.\gamma \in K / A\right)$ is not injective. Hence, the Cousin complex $C(\mathcal{F}, A)^{\bullet}$ is not exact at the zeroth term. The canonical sequence of complexes

$$
0 \longrightarrow{ }^{*} C\left({ }^{*} \mathcal{F}, A\right)^{\bullet} \longrightarrow C(\mathcal{F}, A)^{\bullet} \longrightarrow Q(\mathcal{F}, M)^{\bullet} \longrightarrow 0
$$

therefore shows that $Q(\mathcal{F}, M)^{\bullet}$ is not exact.

Our next aim is to establish that a certain condition is sufficient for the generalized degradation complex of 3.6 to be exact.

Remark and Notation 4.2. Suppose that $(R, \mathfrak{m})$ is ${ }^{*}$ local and that $M \neq 0$. Let $\mathcal{F}=\left(F_{i}\right)_{i \in \mathbb{N}_{0}}$ be a filtration of $\operatorname{Spec}(R)$ which admits $M$ with $\mathfrak{m} \in \partial F_{h}$ (where $h \in \mathbb{N}_{0}$ ) and let ${ }^{*} \mathcal{F}=\left({ }^{*} F_{i}\right)_{i \in \mathbb{N}_{0}}$ be the corresponding ${ }^{*}$ filtration of ${ }^{*} \operatorname{Spec}(R)$, as in Theorem 3.5.

With the terminology of [9, Definition 2.2], we have $h=\mathrm{ht}_{\mathcal{F}} \mathfrak{m}$; also ht ${ }_{M} \mathfrak{m} \leqslant \mathrm{ht}_{\mathcal{F}} \mathfrak{m}=$ $h$ by [9, Lemma 2.3(iv)]. It follows from Grothendieck's Vanishing Theorem for local cohomology that $\left(H_{\mathfrak{m}}^{h}(M)\right)_{\mathfrak{m}}=0$ if $\mathrm{ht}_{M} \mathfrak{m}<h$. Since the local cohomology module $H_{\mathfrak{m}}^{h}(M)$ is $G$-graded, it follows from the $G$-graded analogue of [6, Lemma 2.2(iii)] that the natural homomorphism $\mu_{H_{\mathfrak{m}}^{h}(M)}: H_{\mathfrak{m}}^{h}(M) \longrightarrow\left(H_{\mathfrak{m}}^{h}(M)\right)_{\mathfrak{m}}$ is injective. Therefore, $H_{\mathfrak{m}}^{h}(M)=0$ if $\mathrm{ht}_{M} \mathfrak{m}<h$.

Next, $\operatorname{Supp}\left(H_{\mathfrak{m}}^{h}(M)\right) \subseteq \operatorname{Var}(\mathfrak{m})$. Set $F_{0}^{\prime}=\left\{\mathfrak{p} \in \operatorname{Spec}(R): \mathfrak{p}^{*}=\mathfrak{m}\right\}$, and $F_{i}^{\prime}=\{\mathfrak{p} \in$ $\operatorname{Spec}(R): \mathfrak{p}^{*}=\mathfrak{m}$ and $\left.\operatorname{ht}\left(\mathfrak{p} / \mathfrak{p}^{*}\right) \geqslant i\right\}$ for all $i \in \mathbb{N}$. Then

$$
\operatorname{Spec}(R) \supseteq F_{0}^{\prime} \supseteq F_{1}^{\prime} \supseteq \cdots \supseteq F_{i}^{\prime} \supseteq \cdots,
$$

and $\operatorname{Supp}\left(H_{\mathfrak{m}}^{h}(M)\right) \subseteq F_{0}^{\prime}$. (Note that, by [5, Theorem 1.2.3], as $R$ is $G$-graded, $0 \leqslant$ $\operatorname{ht}\left(\mathfrak{p} / \mathfrak{p}^{*}\right) \leqslant \operatorname{rank}(G)$ for each $\mathfrak{p} \in \operatorname{Spec}(R)$, and so $F_{i}^{\prime}=\emptyset$ for all $i>\operatorname{rank}(G)$.) Therefore, the family $\mathcal{F}^{\prime}=\left(F_{i}^{\prime}\right)_{i \in \mathbb{N}_{0}}$ provides a filtration of $\operatorname{Spec}(R)$ that admits $H_{\mathfrak{m}}^{h}(M)$, so that we can construct the Cousin complex $C\left(\mathcal{F}^{\prime}, H_{\mathfrak{m}}^{h}(M)\right)^{\bullet}$ as in [8, Definition 1.3]. Set $n:=\operatorname{rank}(G)$. An application of [9, Corollary 2.6] shows that the Cousin complex $C\left(\mathcal{F}^{\prime}, H_{\mathfrak{m}}^{h}(M)\right)^{\bullet}$ has the form

$$
\begin{aligned}
0 \longrightarrow H_{\mathfrak{m}}^{h}(M) \longrightarrow\left(H_{\mathfrak{m}}^{h}(M)\right)_{\mathfrak{m}} \longrightarrow \bigoplus_{\mathfrak{q} \in \partial F_{1}^{\prime}}\left(H_{\mathfrak{q}}^{1}\left(H_{\mathfrak{m}}^{h}(M)\right)\right)_{\mathfrak{q}} \longrightarrow \cdots \\
\longrightarrow \bigoplus_{\mathfrak{q} \in \partial F_{n}^{\prime}}\left(H_{\mathfrak{q}}^{n}\left(H_{\mathfrak{m}}^{h}(M)\right)\right)_{\mathfrak{q}} \longrightarrow 0
\end{aligned}
$$


In the next lemma we shall show that the Cousin complex $C\left(\mathcal{F}^{\prime}, H_{\mathfrak{m}}^{h}(M)\right)^{\bullet}$ is exact. Henceforth in this paper we shall, for convenience, use the notation $H_{\mathfrak{m}}^{h}(M)_{\mathfrak{m}}$ and $H_{\mathfrak{p}}^{i}\left(H_{\mathfrak{m}}^{h}(M)\right)_{\mathfrak{p}}$ to denote $\left(H_{\mathfrak{m}}^{h}(M)\right)_{\mathfrak{m}}$ and $\left(H_{\mathfrak{p}}^{i}\left(H_{\mathfrak{m}}^{h}(M)\right)\right)_{\mathfrak{p}}$, respectively, for $i, h \in \mathbb{N}_{0}$ and $\mathfrak{p}, \mathfrak{m} \in \operatorname{Spec}(R)$.

Lemma 4.3. Let the situation and notation be as in 4.2. Then the Cousin complex $C\left(\mathcal{F}^{\prime}, H_{\mathfrak{m}}^{h}(M)\right)^{\bullet}$ is exact.

Proof. Set $H:=H_{\mathfrak{m}}^{h}(M)$. Clearly, we can assume that $H \neq 0$. Then $\mathrm{ht}_{M} \mathfrak{m}=\mathrm{ht}_{\mathcal{F}} \mathfrak{m}=$ $h$ and Ass $H=\{\mathfrak{m}\}$, since $H$ is $\mathfrak{m}$-torsion and $G$-graded. Thus, $\operatorname{Supp}(H)=F_{0}^{\prime}$ and $\mathcal{F}^{\prime}$ is the $H$-height filtration of $\operatorname{Spec}(R)$ (see [8, Examples 1.2]); therefore, $C\left(\mathcal{F}^{\prime}, H\right)^{\bullet}$ is just the Cousin complex $C(H)^{\bullet}$ of $[\mathbf{7}, \S 2]$. Write $C(H)^{\bullet}$ as

$$
0 \stackrel{f^{-2}}{\longrightarrow} H \stackrel{f^{-1}}{\longrightarrow} H^{0} \stackrel{f^{0}}{\longrightarrow} H^{1} \longrightarrow \cdots \longrightarrow H^{i} \stackrel{f^{i}}{\longrightarrow} H^{i+1} \longrightarrow \cdots .
$$

Suppose that $C(H)^{\bullet}$ is not exact, let $v$ be the least $i \in \mathbb{N}_{0}$ such that $C(H)^{\bullet}$ is not exact at $H^{i-1}$ (where we interpret $H^{-1}$ as $H$ ), and let $\mathfrak{a}$ be the annihilator of a non-zero element of the $(v-1)$ th cohomology module of $C(H)^{\bullet}$. Then $\operatorname{Var}(\mathfrak{a}) \subseteq F_{v+1}^{\prime}($ by $[\mathbf{7}, \S 2.7($ vii) $])$, and one can use the 'partially exact Cousin complex argument' of $[\mathbf{7}$, Lemma (4.6)] to see that $\operatorname{Ext}_{R}^{i}(R / \mathfrak{a}, H)=0$ for $i=0, \ldots, v-1$ and $\operatorname{Ext}_{R}^{v}(R / \mathfrak{a}, H) \neq 0$. Since $H$ can be considered as a direct limit of its finitely generated $G$-graded submodules, it follows that there exists a finitely generated $G$-graded submodule $L$ of $H$ such that $\operatorname{Ext}_{R}^{v}(R / \mathfrak{a}, L) \neq 0$.

Since $\operatorname{Ass}(L)=\{\mathfrak{m}\}$, it follows from [5, Corollary 1.2.4] that $L$ is a Cohen-Macaulay $R$-module, so that $\operatorname{Ext}_{R}^{v}(R / \mathfrak{a}, L)=0$ since $\operatorname{Var}(\mathfrak{a}) \subseteq F_{v+1}^{\prime}$. This contradiction completes the proof.

Lemma 4.4. For all $i, j \in \mathbb{N}_{0}$, the $j$ th term in the Cousin complex $C\left(\mathcal{F}^{\prime}, H_{\mathfrak{m}}^{h}(M)\right)^{\bullet}$ of 4.2 satisfies

$$
\operatorname{supp}_{R}^{i}\left(\bigoplus_{\mathfrak{p} \in \partial F_{j}^{\prime}} H_{\mathfrak{p}}^{j}\left(H_{\mathfrak{m}}^{h}(M)\right)_{\mathfrak{p}}\right) \subseteq \partial F_{j}^{\prime} .
$$

Proof. It is enough to show that, for each $\mathfrak{p} \in \partial F_{j}^{\prime}, \operatorname{supp}_{R}^{i}\left(H_{\mathfrak{p}}^{j}\left(H_{\mathfrak{m}}^{h}(M)\right)_{\mathfrak{p}}\right) \subseteq\{\mathfrak{p}\}$ (because the functor $\operatorname{Ext}_{R_{\mathfrak{p}}}^{i}(k(\mathfrak{p}), \cdot)$ (on the category of all $R_{\mathfrak{p}}$-modules and $R_{\mathfrak{p}}$-homomorphisms) commutes with direct limits).

Set $L:=H_{\mathfrak{p}}^{j}\left(H_{\mathfrak{m}}^{h}(M)\right)$. Since $L$ is $\mathfrak{p}$-torsion, it is clear that $\operatorname{Supp}_{R_{\mathfrak{p}}}\left(L_{\mathfrak{p}}\right) \subseteq\left\{\mathfrak{p} R_{\mathfrak{p}}\right\}$. Hence, by Lemma 2.2(ii):

$$
\begin{aligned}
\operatorname{supp}_{R}^{i}\left(L_{\mathfrak{p}}\right) & =\left\{\mathfrak{q} \in \operatorname{Spec}(R): \mathfrak{q} \subseteq \mathfrak{p} \text { and } \mathfrak{q} R_{\mathfrak{p}} \in \operatorname{supp}_{R_{\mathfrak{p}}}^{i}\left(L_{\mathfrak{p}}\right)\right\} \\
& \subseteq\left\{\mathfrak{q} \in \operatorname{Spec}(R): \mathfrak{q} \subseteq \mathfrak{p} \text { and } \mathfrak{q} R_{\mathfrak{p}} \in \operatorname{Supp}_{R_{\mathfrak{p}}}\left(L_{\mathfrak{p}}\right)\right\} \\
& \subseteq\{\mathfrak{p}\} .
\end{aligned}
$$


Proposition 4.5. Let the situation and notation be as in 4.2 and 4.3. Then

$$
\operatorname{supp}^{i}\left(H_{\mathfrak{m}}^{h}(M)_{\mathfrak{m}} / H_{\mathfrak{m}}^{h}(M)\right) \subseteq \bigcup_{j=1}^{i+1} \partial F_{j}^{\prime}
$$

for all $i \in \mathbb{N}_{0}$.

Proof. With the notation of 4.2 , the Cousin complex $C\left(\mathcal{F}^{\prime}, H_{\mathfrak{m}}^{h}(M)\right)^{\bullet}$ is exact, by Lemma 4.3. It therefore follows from the description of that Cousin complex given in 4.2 that there is an exact sequence

$$
\begin{aligned}
& 0 \longrightarrow H_{\mathfrak{m}}^{h}(M)_{\mathfrak{m}} / H_{\mathfrak{m}}^{h}(M) \stackrel{g_{0}}{\longrightarrow} \bigoplus_{\mathfrak{p} \in \partial F_{1}^{\prime}} H_{\mathfrak{p}}^{1}\left(H_{\mathfrak{m}}^{h}(M)\right)_{\mathfrak{p}} \stackrel{g_{1}}{\longrightarrow} \cdots \\
& \bigoplus_{\mathfrak{p} \in \partial F_{i}^{\prime}} H_{\mathfrak{p}}^{i}\left(H_{\mathfrak{m}}^{h}(M)\right)_{\mathfrak{p}} \stackrel{g_{i}}{\longrightarrow} \cdots
\end{aligned}
$$

Let $K_{j}:=\operatorname{Ker} g_{j+1}$ for all $j \in \mathbb{N}_{0}$, so that $K_{0} \cong H_{\mathfrak{m}}^{h}(M)_{\mathfrak{m}} / H_{\mathfrak{m}}^{h}(M)$. Let $i \in \mathbb{N}_{0}$. Use Lemma 2.2(v) (and the interpretation of supp ${ }^{-1}$ given therein), in conjunction with the short exact sequences obtainable from the last-displayed long exact sequence, to see that

$$
\operatorname{supp}^{i-j}\left(K_{j}\right) \subseteq \operatorname{supp}^{i-j}\left(\bigoplus_{\mathfrak{p} \in \partial F_{j+1}^{\prime}} H_{\mathfrak{p}}^{j+1}\left(H_{\mathfrak{m}}^{h}(M)\right)_{\mathfrak{p}}\right) \bigcup \operatorname{supp}^{i-j-1}\left(K_{j+1}\right)
$$

for all $j=0, \ldots, i$. Since $K_{0} \cong H_{\mathfrak{m}}^{h}(M)_{\mathfrak{m}} / H_{\mathfrak{m}}^{h}(M)$, it follows from these inclusions that

$$
\operatorname{supp}^{i}\left(H_{\mathfrak{m}}^{h}(M)_{\mathfrak{m}} / H_{\mathfrak{m}}^{h}(M)\right) \subseteq \bigcup_{j=0}^{i} \operatorname{supp}^{i-j}\left(\bigoplus_{\mathfrak{p} \in \partial F_{j+1}^{\prime}} H_{\mathfrak{p}}^{j+1}\left(H_{\mathfrak{m}}^{h}(M)\right)_{\mathfrak{p}}\right),
$$

and the claim therefore follows from Lemma 4.4.

Remark 4.6. Let the situation and notation be as in 4.2. Then, by Proposition 4.5 and the construction of the filtration $\mathcal{F}^{\prime}$, we have

$$
\begin{aligned}
\operatorname{supp}^{i}\left(H_{\mathfrak{m}}^{h}(M)_{\mathfrak{m}} / H_{\mathfrak{m}}^{h}(M)\right) & \subseteq\left\{\mathfrak{q} \in \operatorname{Spec}(R): \mathfrak{q}^{*}=\mathfrak{m} \text { and } 1 \leqslant \mathrm{ht}\left(\mathfrak{q} / \mathfrak{q}^{*}\right) \leqslant i+1\right\} \\
& =\left\{\mathfrak{q} \in \operatorname{Spec}(R) \backslash{ }^{*} \operatorname{Spec}(R): \mathfrak{q}^{*}=\mathfrak{m}, \operatorname{ht}\left(\mathfrak{q} / \mathfrak{q}^{*}\right) \leqslant i+1\right\}
\end{aligned}
$$

for all $i \in \mathbb{N}_{0}$.

The following corollary is now an immediate consequence of Theorem 3.5(v) and Proposition 4.5.

Corollary 4.7. Suppose that $(R, \mathfrak{m})$ is ${ }^{*}$ local. Let $\mathcal{F}=\left(F_{i}\right)_{i \in \mathbb{N}_{0}}$ be a filtration of $\operatorname{Spec}(R)$ which admits $M$, and suppose that $\mathfrak{m} \in \partial F_{h}$. Let

$$
\Psi(\mathcal{F}, M)=\left(\psi^{i}\right)_{i \geqslant-2}:{ }^{*} C\left({ }^{*} \mathcal{F}, M\right)^{\bullet} \longrightarrow C(\mathcal{F}, M)^{\bullet}
$$


be the chain map of Theorem 3.5. Then, for each $i \in \mathbb{N}_{0}$,

$$
\begin{aligned}
\operatorname{supp}^{i}\left(\left(\operatorname{Coker} e^{h-2}\right)_{\mathfrak{m}} / \psi^{h}\left(\operatorname{Coker} d^{h-2}\right)\right) & \\
& \subseteq\left\{\mathfrak{q} \in \operatorname{Spec}(R) \backslash{ }^{*} \operatorname{Spec}(R): \mathfrak{q}^{*}=\mathfrak{m}, \operatorname{ht}\left(\mathfrak{q} / \mathfrak{q}^{*}\right) \leqslant i+1\right\} .
\end{aligned}
$$

We recall the following definition from the Introduction of $[\mathbf{9}]$.

Definition 4.8. Let $L$ be a module over the commutative Noetherian ring $A^{\prime}$. Let $\mathcal{F}^{\prime}=\left(F_{i}^{\prime}\right)_{i \in \mathbb{N}_{0}}$ be a filtration of $\operatorname{Spec}\left(A^{\prime}\right)$ which admits $L$, and let $C\left(\mathcal{F}^{\prime}, L\right)^{\bullet}$ be the Cousin complex

$$
0 \stackrel{c^{-2}}{\longrightarrow} L \stackrel{c^{-1}}{\longrightarrow} L^{0} \stackrel{c^{0}}{\longrightarrow} L^{1} \longrightarrow \cdots \longrightarrow L^{i} \stackrel{c^{i}}{\longrightarrow} L^{i+1} \longrightarrow \cdots
$$

for $L$ with respect to $\mathcal{F}^{\prime}$. Then we say that a prime ideal $\mathfrak{p}$ of $A^{\prime}$ is significant, or of significance, for $C\left(\mathcal{F}^{\prime}, L\right) \bullet$ if there exists $i \in \mathbb{N}_{0}$ for which $\mathfrak{p} \in \partial F_{i}^{\prime}$ and the direct summand $\left(\text { Coker } c^{i-2}\right)_{\mathfrak{p}}$ of the term $L^{i}$ is non-zero; otherwise, we say that $\mathfrak{p}$ is insignificant, or of no significance, for $C\left(\mathcal{F}^{\prime}, L\right)^{\bullet}$.

Lemma 4.9. Let the situation and notation be as in Theorem 3.5 and 3.6, and let

$$
Q(\mathcal{F}, M)^{\bullet}: 0 \longrightarrow Q^{0} \stackrel{f^{0}}{\longrightarrow} Q^{1} \longrightarrow \cdots \longrightarrow Q^{i} \stackrel{f^{i}}{\longrightarrow} Q^{i+1} \longrightarrow \cdots
$$

be the generalized degradation complex of $M$ with respect to $\mathcal{F}$ of 3.6. Then, for all $i, h \in \mathbb{N}_{0}$,

$$
\begin{array}{r}
\operatorname{supp}_{R}^{i}\left(Q^{h}\right) \subseteq\left\{\mathfrak{q} \in \operatorname{Supp}(M) \backslash{ }^{*} \operatorname{Supp}(M): \mathfrak{q} \in \partial F_{h} \text { or } \mathfrak{q}^{*} \in \partial F_{h} \text { with } \mathfrak{q}^{*}\right. \text { significant } \\
\text { for } \left.C(\mathcal{F}, M)^{\bullet} \text { and } \operatorname{ht}\left(\mathfrak{q} / \mathfrak{q}^{*}\right) \leqslant i+1\right\} .
\end{array}
$$

Proof. Let $i, h \in \mathbb{N}_{0}$. With the notation of 3.6, we have $Q^{h} \cong G^{h} \bigoplus U^{h}$, where

$$
\begin{gathered}
G^{h}:=\bigoplus_{\mathfrak{p} \in \partial F_{h} \cap^{*} \operatorname{Supp}(M)} Q_{R}^{h}(\mathfrak{p}), \\
Q_{R}^{h}(\mathfrak{p})=\left(\operatorname{Coker} e^{h-2}\right)_{\mathfrak{p}} / \psi^{h}\left(\left(\operatorname{Coker} d^{h-2}\right)_{(\mathfrak{p})}\right) \text { for all } \mathfrak{p} \in{ }^{*} \operatorname{Supp}(M) \cap \partial F_{h}, \text { and } \\
U^{h}:=\bigoplus_{\mathfrak{p} \in \partial F_{h} \cap \operatorname{Supp}(M) \backslash * \operatorname{Supp}(M)}\left(\operatorname{Coker} e^{h-2}\right)_{\mathfrak{p}} .
\end{gathered}
$$

(We have made use of $[\mathbf{8}$, Corollary 1.5] here.) Note that

$$
\operatorname{supp}_{R}^{i}\left(Q^{h}\right)=\operatorname{supp}_{R}^{i}\left(G^{h}\right) \cup \operatorname{supp}_{R}^{i}\left(U^{h}\right) .
$$

Since, for each $\mathfrak{p} \in \operatorname{Spec}(R)$, the functor $\operatorname{Ext}_{R_{\mathfrak{p}}}^{i}(k(\mathfrak{p}), \cdot)$ (on the category of all $R_{\mathfrak{p}}$-modules and $R_{\mathfrak{p}}$-homomorphisms) commutes with direct limits, it follows that

$$
\operatorname{supp}_{R}^{i}\left(G^{h}\right)=\bigcup_{\mathfrak{p} \in \partial F_{h} \cap^{*} \operatorname{Supp}(M)} \operatorname{supp}_{R}^{i}\left(Q_{R}^{h}(\mathfrak{p})\right),
$$


and

$$
\operatorname{supp}_{R}^{i}\left(U^{h}\right)=\bigcup_{\mathfrak{p} \in \partial F_{h} \cap \operatorname{Supp}(M) \backslash * \operatorname{Supp}(M)} \operatorname{supp}_{R}^{i}\left(\left(\text { Coker } e^{h-2}\right)_{\mathfrak{p}}\right) .
$$

Now, let $\mathfrak{p} \in \partial F_{h} \cap{ }^{*} \operatorname{Supp}(M)$. Use the notation of 3.6, with the abbreviation $Q_{R}^{i}(\mathfrak{p})$ for $Q_{R}^{i}(\mathfrak{p}, \mathcal{F}, M)$. By 3.6, there is an $R_{(\mathfrak{p})}$-isomorphism

$$
Q_{R}^{i}(\mathfrak{p}, \mathcal{F}, M) \cong Q_{R_{(\mathfrak{p})}}^{i}\left(\mathfrak{p} R_{(\mathfrak{p})}, \mathcal{F}_{(\mathfrak{p})}, M_{(\mathfrak{p})}\right),
$$

and so, by Corollary 4.7, we have

$$
\begin{aligned}
& \operatorname{supp}_{R_{(\mathfrak{p})}}^{i}\left(Q_{R}^{h}(\mathfrak{p})\right) \\
& \quad \subseteq\left\{\mathfrak{Q} \in \operatorname{Supp}_{R_{(\mathfrak{p})}}\left(M_{(\mathfrak{p})}\right) \backslash{ }^{*} \operatorname{Supp}_{R_{(\mathfrak{p})}}\left(M_{(\mathfrak{p})}\right): \mathfrak{Q}^{*}=\mathfrak{p} R_{(\mathfrak{p})}, \operatorname{ht}\left(\mathfrak{Q} / \mathfrak{Q}^{*}\right) \leqslant i+1\right\} .
\end{aligned}
$$

On the other hand, by Definition 2.1 and 3.6 we have

$$
\begin{aligned}
\operatorname{supp}_{R_{(\mathfrak{p})}}^{i}\left(Q_{R}^{h}(\mathfrak{p})\right) & \subseteq \operatorname{supp}_{R_{(\mathfrak{p})}}\left(Q_{R}^{h}(\mathfrak{p})\right) \\
& \subseteq \operatorname{Supp}_{R_{(\mathfrak{p})}}\left(Q_{R}^{h}(\mathfrak{p})\right) \\
& \subseteq\left\{\mathfrak{q} R_{(\mathfrak{p})}: \mathfrak{q} \in \operatorname{Supp}(M) \backslash{ }^{*} \operatorname{Supp}(M) \text { and } \mathfrak{q}^{*}=\mathfrak{p}\right\}
\end{aligned}
$$

We now compare these two bounds for $\operatorname{supp}_{R_{(\mathfrak{p})}}^{i}\left(Q_{R}^{h}(\mathfrak{p})\right)$ to deduce that

$$
\begin{gathered}
\operatorname{supp}_{R_{(\mathfrak{p})}}^{i}\left(Q_{R}^{h}(\mathfrak{p})\right) \subseteq\left\{\mathfrak{q} R_{(\mathfrak{p})} \in \operatorname{Spec}\left(R_{(\mathfrak{p})}\right): \mathfrak{q} \in \operatorname{Supp}(M) \backslash{ }^{*} \operatorname{Supp}(M), \mathfrak{q}^{*}=\mathfrak{p},\right. \\
\text { and } \left.\operatorname{ht}\left(\mathfrak{q} / \mathfrak{q}^{*}\right) \leqslant i+1\right\} .
\end{gathered}
$$

Next, an application of Lemma 2.2(ii) shows that

$$
\operatorname{supp}_{R}^{i}\left(Q^{h}(\mathfrak{p})\right) \subseteq\left\{\mathfrak{q} \in \operatorname{Supp}(M) \backslash{ }^{*} \operatorname{Supp}(M): \mathfrak{q}^{*}=\mathfrak{p} \text { and } \operatorname{ht}\left(\mathfrak{q} / \mathfrak{q}^{*}\right) \leqslant i+1\right\} .
$$

Note that, for a $\mathfrak{p} \in \partial F_{h} \cap{ }^{*} \operatorname{Supp}(M)$, if $\left(\text { Coker } e^{h-2}\right)_{\mathfrak{p}}=0$, then $\operatorname{supp}_{R}^{i}\left(Q_{R}^{h}(\mathfrak{p})\right)=\emptyset$, and if $\left(\text { Coker } e^{h-2}\right)_{\mathfrak{p}} \neq 0$, then $\mathfrak{p}$ is significant for $C(\mathcal{F}, M)^{\bullet}$.

Let $\mathfrak{p} \in \operatorname{Supp}(M) \backslash{ }^{*} \operatorname{Supp}(M)$ with $\mathfrak{p} \in \partial F_{h}$. Then we obtain from Definition 2.1 and $[\mathbf{6}$, Lemma 1.2] that

$$
\begin{aligned}
\operatorname{supp}_{R}^{i}\left(\left(\operatorname{Coker}^{h-2}\right)_{\mathfrak{p}}\right) & \subseteq \operatorname{supp}_{R}\left(\left(\operatorname{Coker} e^{h-2}\right)_{\mathfrak{p}}\right) \\
& \subseteq\left\{\mathfrak{q} \in \operatorname{Spec}(R): \mathfrak{q} \subseteq \mathfrak{p} \text { and } \mathfrak{q} R_{\mathfrak{p}} \in \operatorname{supp}_{R_{\mathfrak{p}}}\left(\left(\operatorname{Coker} e^{h-2}\right)_{\mathfrak{p}}\right)\right\} \\
& \subseteq\left\{\mathfrak{q} \in \operatorname{Spec}(R): \mathfrak{q} \subseteq \mathfrak{p} \text { and } \mathfrak{q} R_{\mathfrak{p}} \in \operatorname{Supp}_{R_{\mathfrak{p}}}\left(\left(\operatorname{Coker} e^{h-2}\right)_{\mathfrak{p}}\right)\right\} \\
& \subseteq\{\mathfrak{p}\} .
\end{aligned}
$$

The result follows.

The next theorem gives a sufficient condition for the generalized degradation complex to be exact. Its statement involves the concept of the $\mathcal{F}$-height, ht $\mathcal{F} \mathfrak{p}$, of a prime ideal $\mathfrak{p}$ of $R$ introduced in [9, Definition 2.2]. 
Theorem 4.10. Let the situation and notation be as in Theorem 3.5 and 3.6, and let

$$
Q(\mathcal{F}, M)^{\bullet}: 0 \longrightarrow Q^{0} \stackrel{f^{0}}{\longrightarrow} Q^{1} \longrightarrow \cdots \longrightarrow Q^{i} \stackrel{f^{i}}{\longrightarrow} Q^{i+1} \longrightarrow \cdots
$$

be the generalized degradation complex of $M$ with respect to $\mathcal{F}$ of 3.6. Suppose that, whenever $\mathfrak{p}$ is a non-G-graded prime ideal in $\operatorname{Supp}(M)$ with $\mathfrak{p}^{*}$ significant for $C(\mathcal{F}, M)^{\bullet}$, then

$$
\operatorname{ht}_{\mathcal{F}} \mathfrak{p}=\operatorname{ht}_{\mathcal{F}} \mathfrak{p}^{*}+\operatorname{ht}\left(\mathfrak{p} / \mathfrak{p}^{*}\right) .
$$

Then $Q(\mathcal{F}, M)^{\bullet}$ is exact.

Proof. The canonical sequence of complexes

$$
0 \longrightarrow{ }^{*} C\left({ }^{*} \mathcal{F}, M\right)^{\bullet} \longrightarrow C(\mathcal{F}, M)^{\bullet} \longrightarrow Q(\mathcal{F}, M)^{\bullet} \longrightarrow 0
$$

induces a long exact sequence,

$$
\begin{aligned}
\cdots & \longrightarrow H^{i}\left({ }^{*} C\left({ }^{*} \mathcal{F}, M\right)^{\bullet}\right) \longrightarrow H^{i}\left(C(\mathcal{F}, M)^{\bullet}\right) \longrightarrow H^{i}\left(Q(\mathcal{F}, M)^{\bullet}\right) \\
& \longrightarrow H^{i+1}\left({ }^{*} C\left({ }^{*} \mathcal{F}, M\right)^{\bullet}\right) \cdots
\end{aligned}
$$

of cohomology modules. Use of this in conjunction with [8, Proposition 1.4(i)] and its $G$-graded analogue shows that, for each $i \in \mathbb{N}_{0}$,

$$
\begin{aligned}
\operatorname{Ass}_{R}\left(H^{i}\left(Q(\mathcal{F}, M)^{\bullet}\right)\right) & \subseteq \operatorname{Supp}\left(H^{i}\left(C(\mathcal{F}, M)^{\bullet}\right)\right) \cup \operatorname{Supp}\left(H^{i+1}\left({ }^{*} C\left({ }^{*} \mathcal{F}, M\right)^{\bullet}\right)\right) \\
& \subseteq F_{i+2} .
\end{aligned}
$$

Let $i, j \in \mathbb{N}_{0}$. It follows from Lemma 4.9 and the hypotheses that

$$
\begin{aligned}
\operatorname{supp}_{R}^{i}\left(Q^{j}\right) & \subseteq\left\{\mathfrak{q} \in \operatorname{Supp}(M) \backslash{ }^{*} \operatorname{Supp}(M): \mathfrak{q} \in \partial F_{j} \text { or ht } \operatorname{ht}_{\mathcal{F}} \leqslant i+j+1\right\} \\
& =\left\{\mathfrak{q} \in \operatorname{Supp}(M) \backslash{ }^{*} \operatorname{Supp}(M): \operatorname{ht}_{\mathcal{F}} \mathfrak{q} \leqslant i+j+1\right\} .
\end{aligned}
$$

Since $H^{0}\left(Q(\mathcal{F}, M)^{\bullet}\right)$ is a submodule of $Q^{0}$, we conclude from Lemma 2.2(i) and (4.2) that

$$
\begin{aligned}
\operatorname{Ass}_{R}\left(H^{0}\left(Q(\mathcal{F}, M)^{\bullet}\right)\right) & \subseteq \operatorname{Ass}_{R}\left(Q^{0}\right)=\operatorname{supp}_{R}^{0}\left(Q^{0}\right) \\
& \subseteq\left\{\mathfrak{p} \in \operatorname{Supp}(M) \backslash{ }^{*} \operatorname{Supp}(M): \operatorname{ht}_{\mathcal{F}} \mathfrak{p} \leqslant 1\right\} \\
& =\left\{\mathfrak{p} \in \operatorname{Supp}(M) \backslash^{*} \operatorname{Supp}(M): \operatorname{ht}_{\mathcal{F}} \mathfrak{p}=1\right\}
\end{aligned}
$$

(There is no non- $G$-graded prime ideal $\mathfrak{p}$ in $\operatorname{Supp}(M)$ with $h_{\mathcal{F}} \mathfrak{p}=0$.) We can now use this in conjunction with (4.1) to deduce that $\operatorname{Ass}_{R}\left(H^{0}\left(Q(\mathcal{F}, M)^{\bullet}\right)\right)=\emptyset$, so that $H^{0}\left(Q(\mathcal{F}, M)^{\bullet}\right)=0$.

We now use an inductive argument, for which it will be convenient to write $Q^{-1}:=0$, and to denote the (zero) homomorphism from $Q^{-1}$ to $Q^{0}$ by $f^{-1}$. Note that Coker $f^{-1}=$ $Q^{0}$; thus, (4.3) shows that

$$
\operatorname{Ass}_{R}\left(\operatorname{Coker} f^{-1}\right)=\operatorname{supp}_{R}^{0}\left(Q^{0}\right) \subseteq\left\{\mathfrak{p} \in \operatorname{Supp}(M) \backslash{ }^{*} \operatorname{Supp}(M): \operatorname{ht}_{\mathcal{F}} \mathfrak{p}=1\right\} .
$$


Suppose, inductively, that $j>0$ and that we have proved that $H^{i}\left(Q(\mathcal{F}, M)^{\bullet}\right)=0$ for all $i<j$. The fact that the complex $Q(\mathcal{F}, M)^{\bullet}$ is exact at $Q^{i}$ for all $i<j$ means that there are canonical exact sequences

$$
\begin{aligned}
& 0 \longrightarrow \text { Coker } f^{j-2} \longrightarrow Q^{j} \longrightarrow \text { Coker } f^{j-1} \longrightarrow 0, \\
& 0 \longrightarrow \text { Coker } f^{j-3} \longrightarrow Q^{j-1} \longrightarrow \text { Coker } f^{j-2} \longrightarrow 0 \text {, } \\
& 0 \longrightarrow \text { Coker } f^{-1} \longrightarrow Q^{1} \longrightarrow \text { Coker } f^{0} \longrightarrow 0 \text {. }
\end{aligned}
$$

We now use these short exact sequences in conjunction with Lemma 2.2(i),(iv) to obtain

$$
\begin{aligned}
& \operatorname{Ass}_{R}\left(\operatorname{Coker} f^{j-1}\right) \subseteq \operatorname{supp}_{R}^{0}\left(Q^{j}\right) \cup \operatorname{supp}_{R}^{1}\left(\operatorname{Coker} f^{j-2}\right), \\
& \operatorname{supp}_{R}^{1}\left(\operatorname{Coker} f^{j-2}\right) \subseteq \operatorname{supp}_{R}^{1}\left(Q^{j-1}\right) \cup \operatorname{supp}_{R}^{2}\left(\text { Coker } f^{j-3}\right), \\
& \vdots \\
& \operatorname{supp}_{R}^{j-1}\left(\operatorname{Coker} f^{0}\right) \subseteq \operatorname{supp}_{R}^{j-1}\left(Q^{1}\right) \cup \operatorname{supp}_{R}^{j}\left(Q^{0}\right) .
\end{aligned}
$$

Therefore,

$$
\operatorname{Ass}_{R}\left(\operatorname{Coker} f^{j-1}\right) \subseteq \operatorname{supp}_{R}^{0}\left(Q^{j}\right) \cup \operatorname{supp}_{R}^{1}\left(Q^{j-1}\right) \cup \cdots \cup \operatorname{supp}_{R}^{j}\left(Q^{0}\right) .
$$

Consequently, it follows from (4.2) that

$$
\operatorname{Ass}_{R}\left(\operatorname{Coker} f^{j-1}\right) \subseteq\left\{\mathfrak{p} \in \operatorname{Supp}(M) \backslash{ }^{*} \operatorname{Supp}(M): \operatorname{ht}_{\mathcal{F}} \mathfrak{p} \leqslant j+1\right\} .
$$

Since $H^{j}\left(Q(\mathcal{F}, M)^{\bullet}\right)$ is a submodule of Coker $f^{j-1}$, we have

$$
\operatorname{Ass}_{R}\left(H^{j}\left(Q(\mathcal{F}, M)^{\bullet}\right)\right) \subseteq\left\{\mathfrak{p} \in \operatorname{Supp}(M) \backslash{ }^{*} \operatorname{Supp}(M): \operatorname{ht}_{\mathcal{F}} \mathfrak{p} \leqslant j+1\right\} .
$$

This, when used in conjunction with (4.1), shows that

$$
\operatorname{Ass}_{R}\left(H^{j}\left(Q(\mathcal{F}, M)^{\bullet}\right)\right)=\emptyset
$$

so that $H^{j}\left(Q(\mathcal{F}, M)^{\bullet}\right)=0$. This completes the inductive step.

When we take, in Theorem 4.10, the filtration $\mathcal{F}$ to be the $M$-height filtration of $\operatorname{Spec}(R)$ (see [8, Examples 1.2]), the Cousin complex $C(\mathcal{F}, M)^{\bullet}$ is just the 'basic' Cousin complex $C(M)^{\bullet}$ of $[\mathbf{7}]$, while the $G$-graded Cousin complex ${ }^{*} C\left({ }^{*} \mathcal{F}, M\right)^{\bullet}$ is nothing other than the Cousin complex ${ }^{*} C(M)^{\bullet}$ for $M$ studied by Goto and Watanabe in [5, Chapter I, $\S 4]$. Since $\operatorname{ht}_{M} \mathfrak{p}=\mathrm{ht}_{M} \mathfrak{p}^{*}+\mathrm{ht}\left(\mathfrak{p} / \mathfrak{p}^{*}\right)$ for each $\mathfrak{p} \in \operatorname{Supp}(M)$ (by [5, Proposition 1.2.2]), we can immediately obtain the $G$-graded analogues of [6, Theorem 3.6 and Corollary 3.7].

Corollary 4.11. Let $M$ be a $G$-graded (not necessarily finitely generated) $R$-module, and let $i$ be an integer with $i \geqslant-1$. Let $C(M)^{\bullet}$ denote the basic Cousin complex of [7], and let ${ }^{*} C(M)^{\bullet}$ denote the G-graded Cousin complex of [5, Chapter I, $\left.\S 4\right]$. 
For this situation, the generalized degradation complex $Q(M)^{\bullet}:=Q(\mathcal{H}(M), M)^{\bullet}$ (where $\mathcal{H}(M)$ denotes the $M$-height filtration of $\operatorname{Spec}(R)$ ) is always exact.

Consequently, $H^{i}\left({ }^{*} C(M)^{\bullet}\right) \cong H^{i}\left(C(M)^{\bullet}\right)$, so that the latter cohomology module inherits a structure as $G$-graded $R$-module, and all its associated primes are $G$-graded; furthermore, $C(M)^{\bullet}$ is exact at its ith term if and only if ${ }^{*} C(M)^{\bullet}$ is exact at its ith term.

Proof. This follows from the comments immediately preceding its statement, together with an argument similar to that used in the proof of [6, Corollary 3.7].

Acknowledgements. M.H.D.A. and R.E. were supported by the Ministry of Culture and Higher Education of the Islamic Republic of Iran.

\section{References}

1. M. P. BRodmann AND R. Y. SharP, Local cohomology: an algebraic introduction with geometric applications (Cambridge University Press, 1998).

2. M. H. Dogani Aghcheghloo, Generalized Hughes complexes and comparison of Cousin complexes in the multigraded case, $\mathrm{PhD}$ thesis, University of Sheffield (1998).

3. R. Enshaei, Modules of generalized fractions and graded rings and modules, $\mathrm{PhD}$ thesis, University of Sheffield (1987).

4. S. Goto And K. Watanabe, On graded rings, I, J. Math. Soc. Japan 30 (1978), 179-213.

5. S. Goto and K. Watanabe, On graded rings, II ( $\mathbb{Z}^{n}$-graded rings), Tokyo J. Math. 1 (1978), 237-261.

6. H. Petzl And R. Y. Sharp, Comparison of graded and ungraded Cousin complexes, Proc. Edinb. Math. Soc. 41 (1998), 289-301.

7. R. Y. Sharp, The Cousin complex for a module over a commutative Noetherian ring, Math. Zeit. 112 (1969), 340-356.

8. R. Y. SHARP, A Cousin complex characterization of balanced big Cohen-Macaulay modules, Q. J. Math. Oxford (2) 33 (1982), 471-485.

9. R. Y. Sharp AND Z. TANG, On the structure of Cousin complexes, J. Math. Kyoto Univ. 33 (1993), 285-297. 